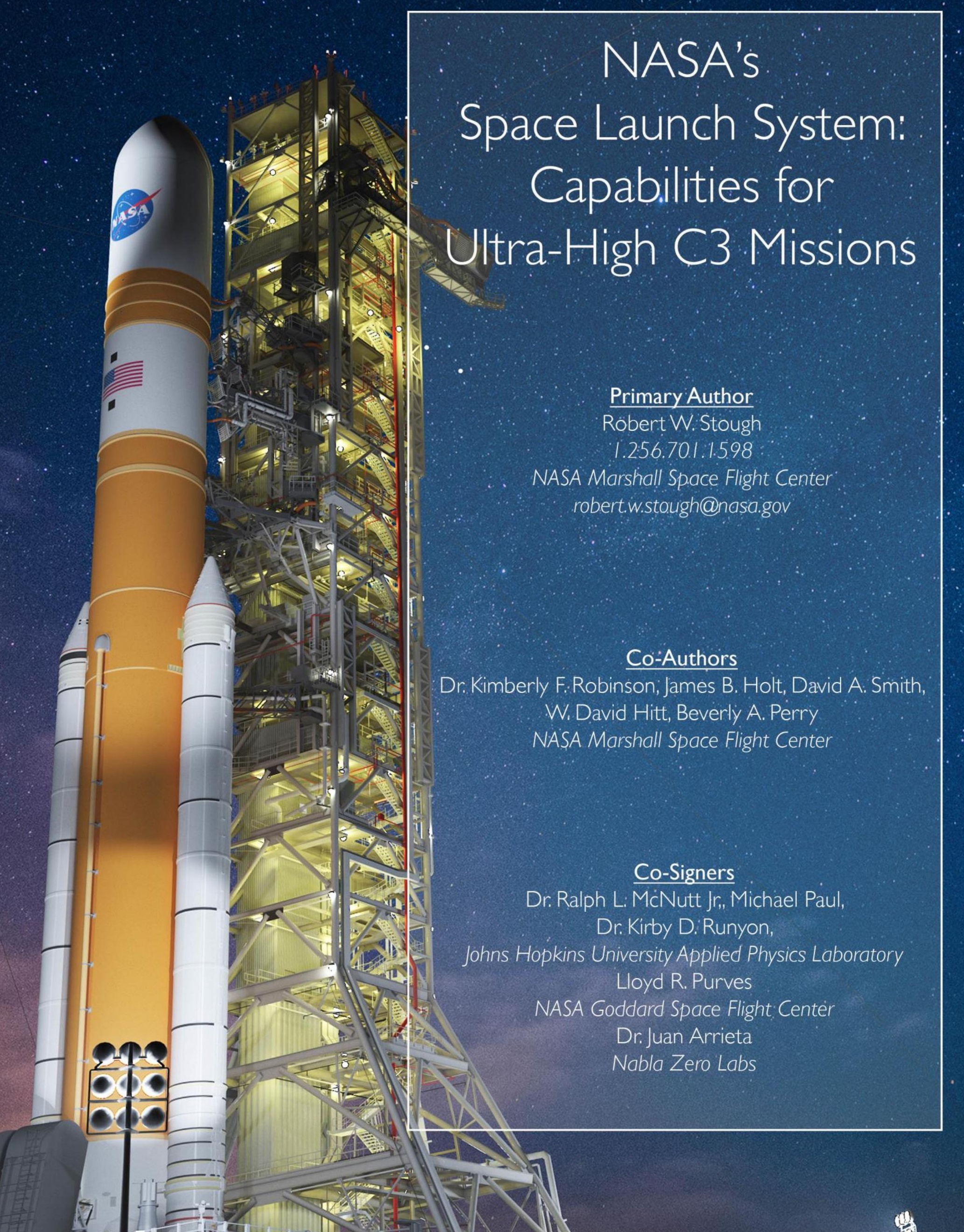




\section{NASA’s Space Launch System: Capabilities for Ultra-High C3 Missions}

A White Paper for the Planetary Science and Astrobiology Decadal Survey 2023-2032

\section{Executive Summary}

- Designed to meet NASA's requirements for human exploration of the Moon, Mars and beyond, the Space Launch System (SLS) vehicle offers unique enhancing and enabling capabilities for a variety of missions, including planetary science, planetary defense and astrobiology.[1, 2]

- Adding commercially available LH2/LOX and/or solid propulsion systems as third and/or fourth stages increases the C3 performance potential of SLS to double the highest-C3 missions ever flown, or enables much greater mass to previously flown trajectories. [3]

- Multiple mission concept studies have shown benefits of utilization of SLS, including Interstellar Probe [4], Neptune Odyssey[5], Pluto Persephone[6]. Enceladus Orbilander [7], and Europa Lander [8]. Studies have also shown benefits of SLS for planetary defense. $[9,10]$

- Today, SLS is making progress toward its initial launch capability and toward both future launches and future capabilities. NASA has issued contracts for SLS hardware for delivery well into the 2030s to support manufacture of evolved versions of the vehicle.

\section{SLS Overview}

Together with the Orion crew vehicle and the Exploration Ground Systems Program revitalizing Kennedy Space Center (KSC), SLS is a cornerstone of the Artemis program, NASA's effort to land the first woman and the next man on the lunar surface. The Artemis I flight of SLS and Orion is the beginning of a $21_{\mathrm{st}}$-century exploration endeavor with the goal of sustained human presence on and around the Moon supporting scientific goals and ultimately the first human steps

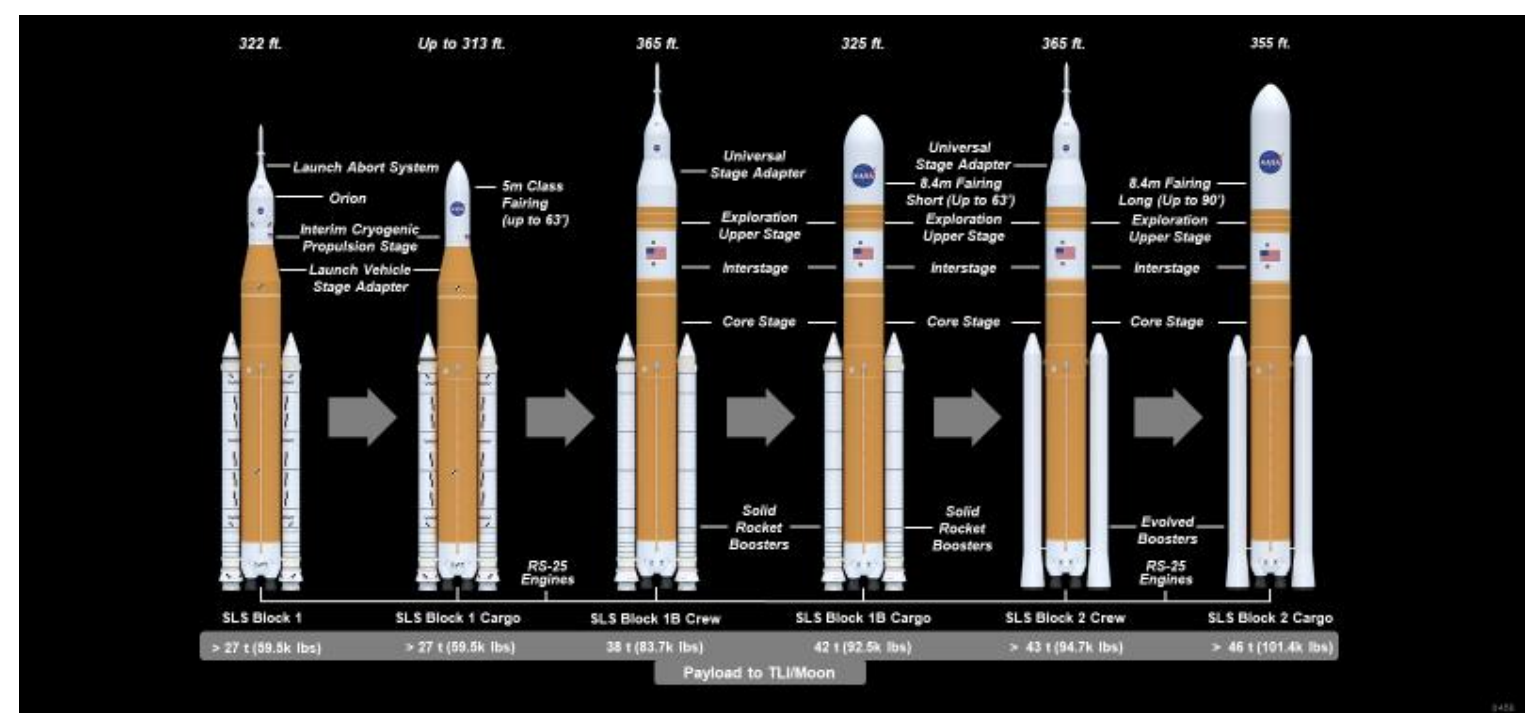

Figure 1. SLS evolution path 
on Mars. For the first crewed landing mission, Artemis III, SLS will launch crew in Orion to a commercially developed Human Landing System waiting in lunar orbit, which will then descend to the lunar surface.

SLS is an evolving capability which will fly in a series of three increasingly powerful configurations, each of which will be available in crew and cargo variants. (Figure 1) The initial Block 1 vehicle can be flown in a cargo configuration with a commercially available $5 \mathrm{~m}$ fairing. The second configuration of SLS, the Block 1B vehicle, can be flown with an $8.4 \mathrm{~m}$ fairing up to $27.4 \mathrm{~m}$ in length. In its crewed configuration, Block $1 \mathrm{~B}$ can carry large payloads (up to $10 \mathrm{t}$ ) comanifested with Orion, in a universal stage adapter (USA) offering as much volume as an industry-standard $5 \mathrm{~m}$ fairing. The ultimate configuration of the vehicle, Block 2, offers the potential to fly larger $10 \mathrm{~m}$ fairings (Figure 2). In these baseline configurations, SLS offers significant performance for a wide range of missions. The Block 1 vehicle is capable of launching 27 metric tons (t) to trans-lunar injection (TLI), approximately $20 \mathrm{t}$ to Mars, and $6 \mathrm{t}$ direct to Jupiter. The Block 2 vehicle offers the ability to deliver greater than $46 \mathrm{t}$ to TLI, greater than 35 t to Mars and approximately 8 t direct to Jupiter (Figure 3). More information about SLS performance and environments can be found in the "Space Launch System (SLS) Mission Planner's Guide." [1]

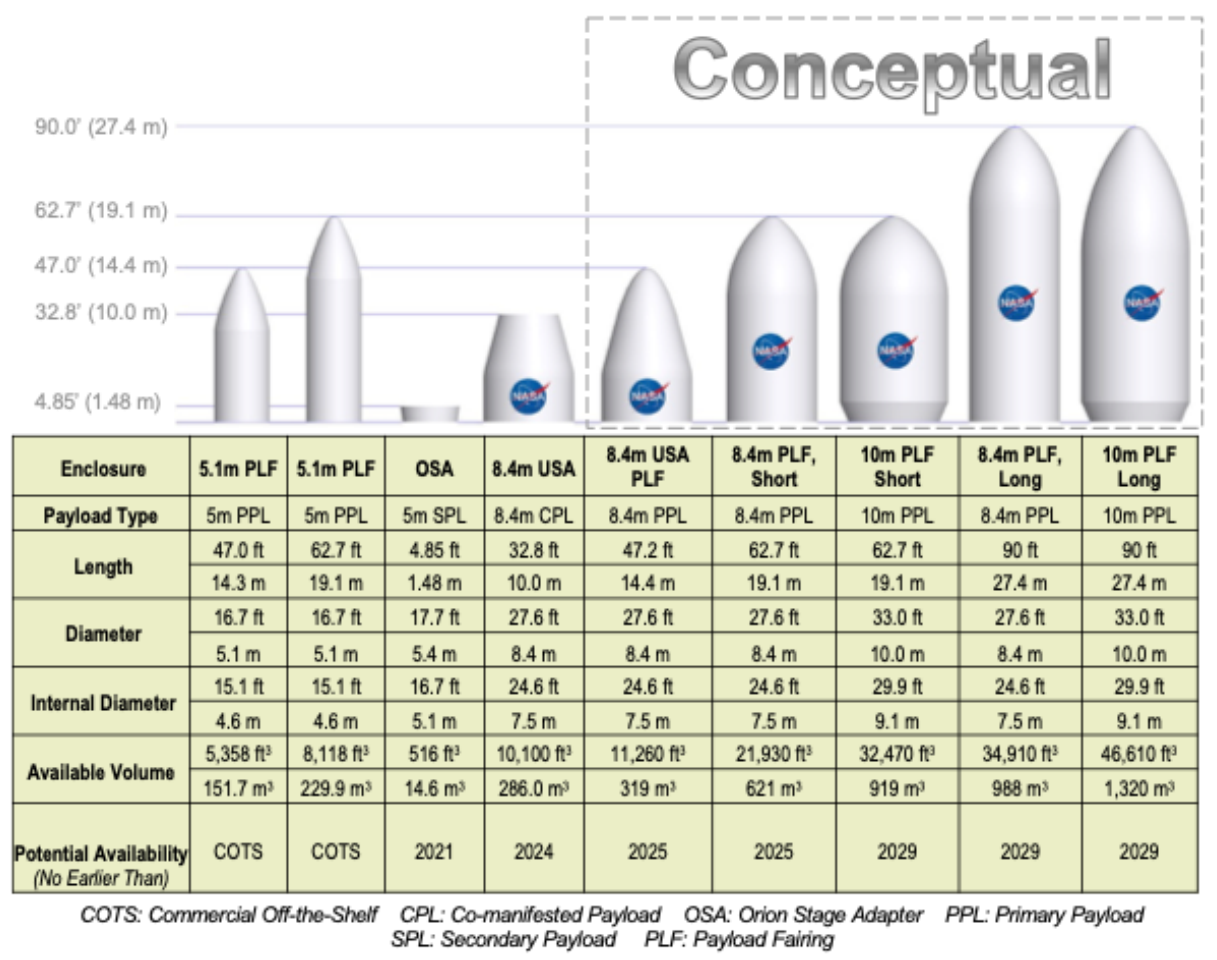

Figure 2. Notional range of payload fairings available on SLS configurations

From its first flight, SLS will support science missions; the Artemis 1 mission is planned to carry 13 CubeSats as secondary payloads, of which six can be classified as planetary missions (five to study the Moon and one to study a near-Earth asteroid). [2] As the Artemis program proceeds with ongoing lunar flights, there may be the opportunity for more such secondary payloads. Also, the individual payloads could be larger and more capable (e.g., ESPA-class smallsats), 
particularly when the Block 1B SLS is developed. In addition, the Artemis missions themselves will contribute to lunar science, with NASA's Science Mission Directorate outlining science goals for the crewed landings.

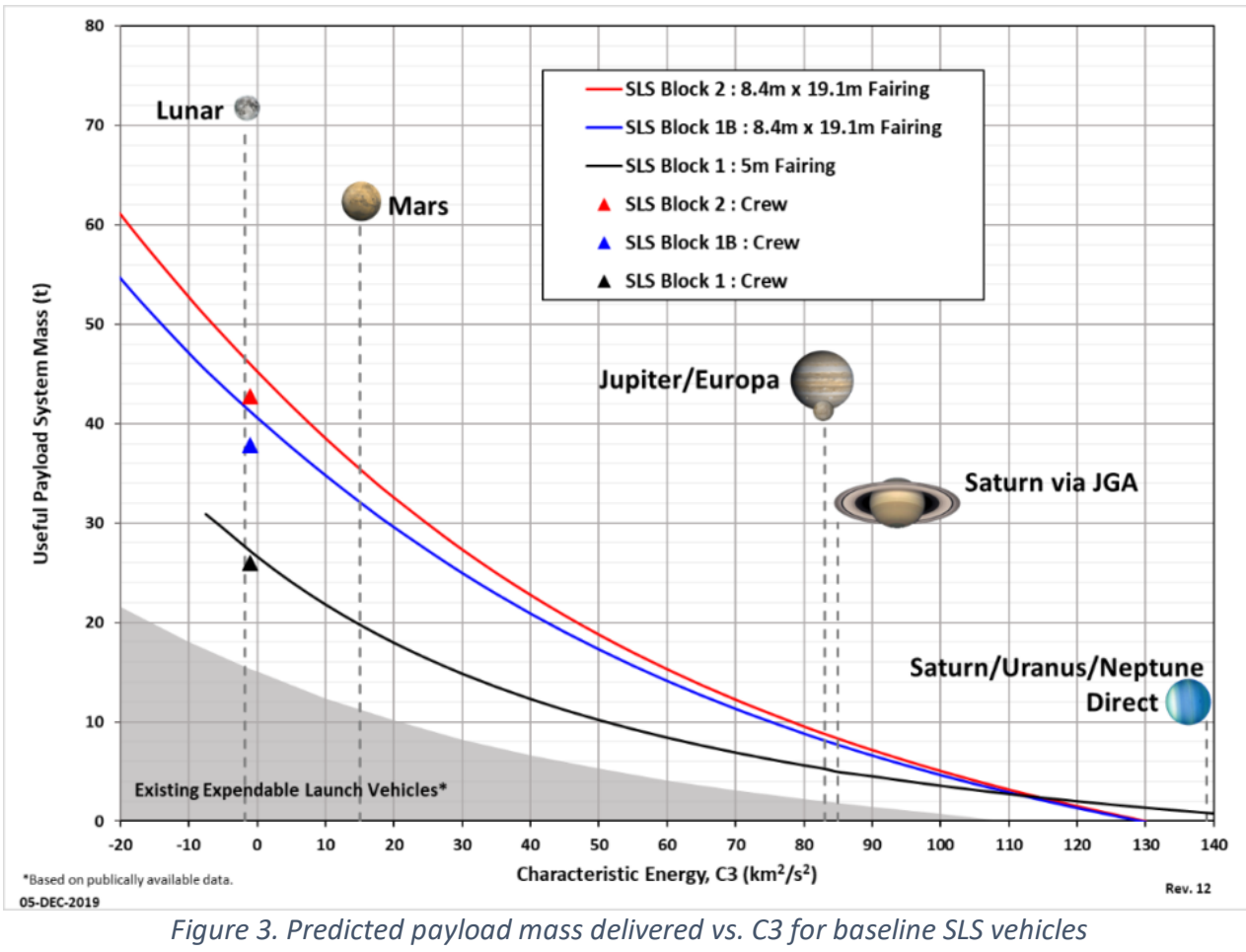

For dedicated science missions, a large launch vehicle such as SLS offers numerous benefits. All other things being equal, the relationship between propellant mass and dry mass as launch vehicle size increases reduces cost-per-kilogram, which is why the Saturn V, despite being the most expensive launch vehicle, was considered to have the lowest cost $/ \mathrm{kg}$ of payload to orbit; whereas the Pegasus, the smallest and least expensive launch vehicle, had the highest cost/kg of payload to orbit. Also, a cargo SLS launch offers a unique combination - the reliability of a human-rated launch vehicle, without the requirements of a crewed launch. While SLS can be used to launch ultra-large payloads, that performance can be traded to launch a smaller payload to high departure energies. Doing so can eliminate gravity assists from the mission profile, increasing launch windows, decreasing the cost of getting mission reliability over lengthy cruise period, reducing operation costs during long cruise period, and expediting return of science results. For these and other reasons SLS has been engaged to provide performance and other supporting data for multiple planetary missions being studied, including Europa Clipper and Mars Sample Return.

\section{SLS for Ultra-High C3 Missions}

Because SLS has the capability to launch strategic science missions in addition to serving as the backbone for Artemis lunar missions, the SLS Program, through its Spacecraft/Payload Integration \& Evolution (SPIE) office, partnered with the Advanced Concepts Office (ACO) at NASA's Marshall Space Flight Center (MSFC), where the program is managed, to perform studies to predict $\mathrm{C} 3$ performance of the vehicle with the addition of commercially available third and fourth stages. 
The SLS Block 2 configuration is the primary focus of the SPIE/ACO analysis. During this study, the approach to predicting the mass delivered targeted a balanced approach. [2] The approach assumed mean vehicle mass, nominal liquid engine performance, and low performance booster. This approach resulted in conservative performance predictions that are below the nominal performance predictions. This study also assumed encapsulation of the additional stages under an $8.4 \mathrm{~m}$ fairing $19.1 \mathrm{~m}$ in length (Figure 2) along with the spacecraft/payload. Encapsulating the additional stage(s) under this fairing produces time and cost savings as compared to certifying a new SLS vehicle configuration. For the study, the stages analyzed are LH2/LOX or solid propellant stages that are currently in production and flying (Table 1). In addition to modeling the performance of existing stages, the stage-to-stage adapters were sized as part of this study. After applying the ground rules described in the previous section and removing the manager's reserve, the predicted high C3 performance is shown in figures 4 through 6 .

\begin{tabular}{|c|l|l|l|}
\hline Stage \# & Stage & Manufacturer & \multicolumn{1}{|c|}{ Propellant Type } \\
\hline $\mathbf{3}$ & CASTOR 30B & NGIS & Hydroxyl-terminated polybutadiene (HTPB) \\
\hline $\mathbf{3}$ & CASTOR 30XL & NGIS & Hydroxyl-terminated polybutadiene (HTPB) \\
\hline $\mathbf{3}$ & Orion 5OXL & NGIS & Hydroxyl-terminated polybutadiene (HTPB) \\
\hline $\mathbf{3}$ & Centaur & ULA & Liquid oxygen (LOX) / Liquid Hydrogen (LH2) \\
\hline $\mathbf{3}$ & Centaur D & ULA & Liquid oxygen (LOX) / Liquid Hydrogen (LH2) \\
\hline $\mathbf{3}$ or $\mathbf{4}$ & Star 48 BV & NGIS & Hydroxyl-terminated polybutadiene (HTPB) \\
\hline $\mathbf{3}$ or $\mathbf{4}$ & Star 48 GXV & NGIS & Hydroxyl-terminated polybutadiene (HTPB) \\
\hline
\end{tabular}

Table 1. List of third and fourth stages assessed

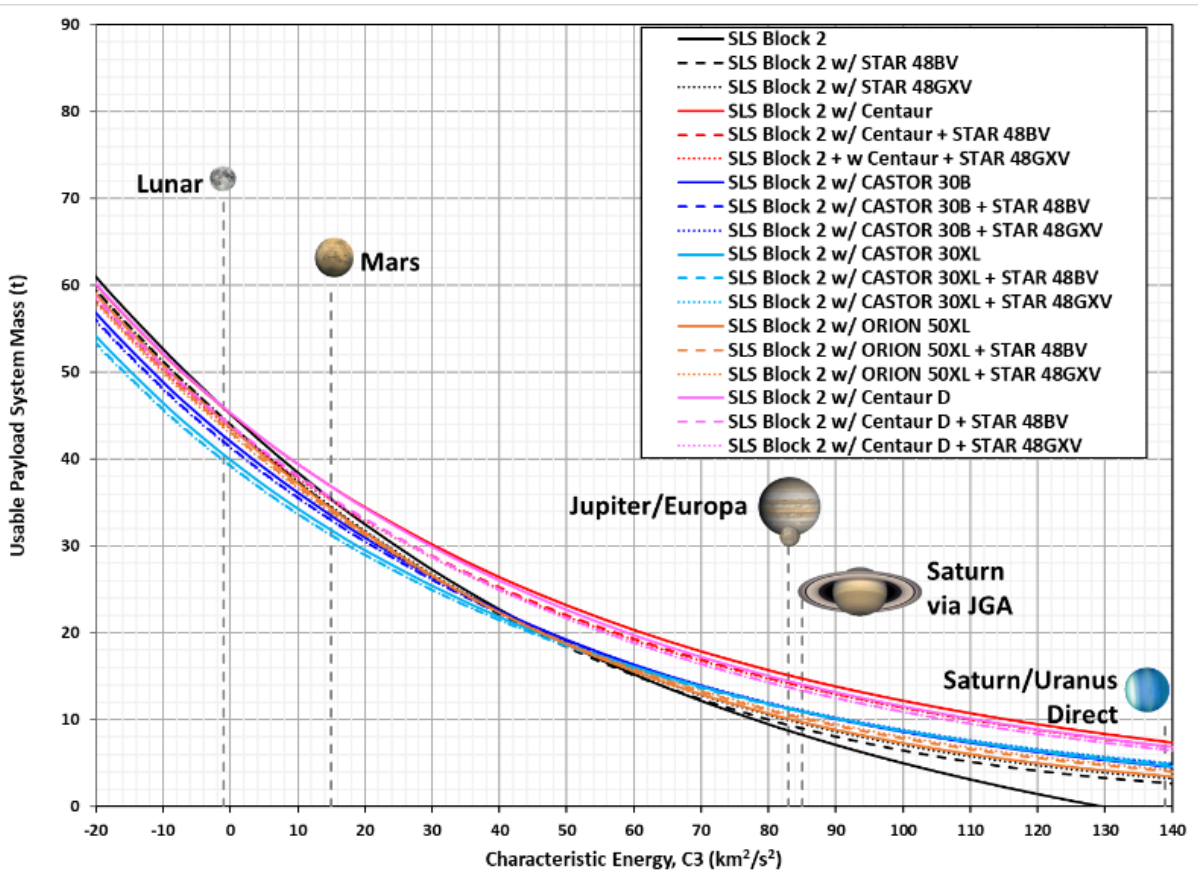

Figure 4. Low C3 range for SLS Block 2 with additional stages 

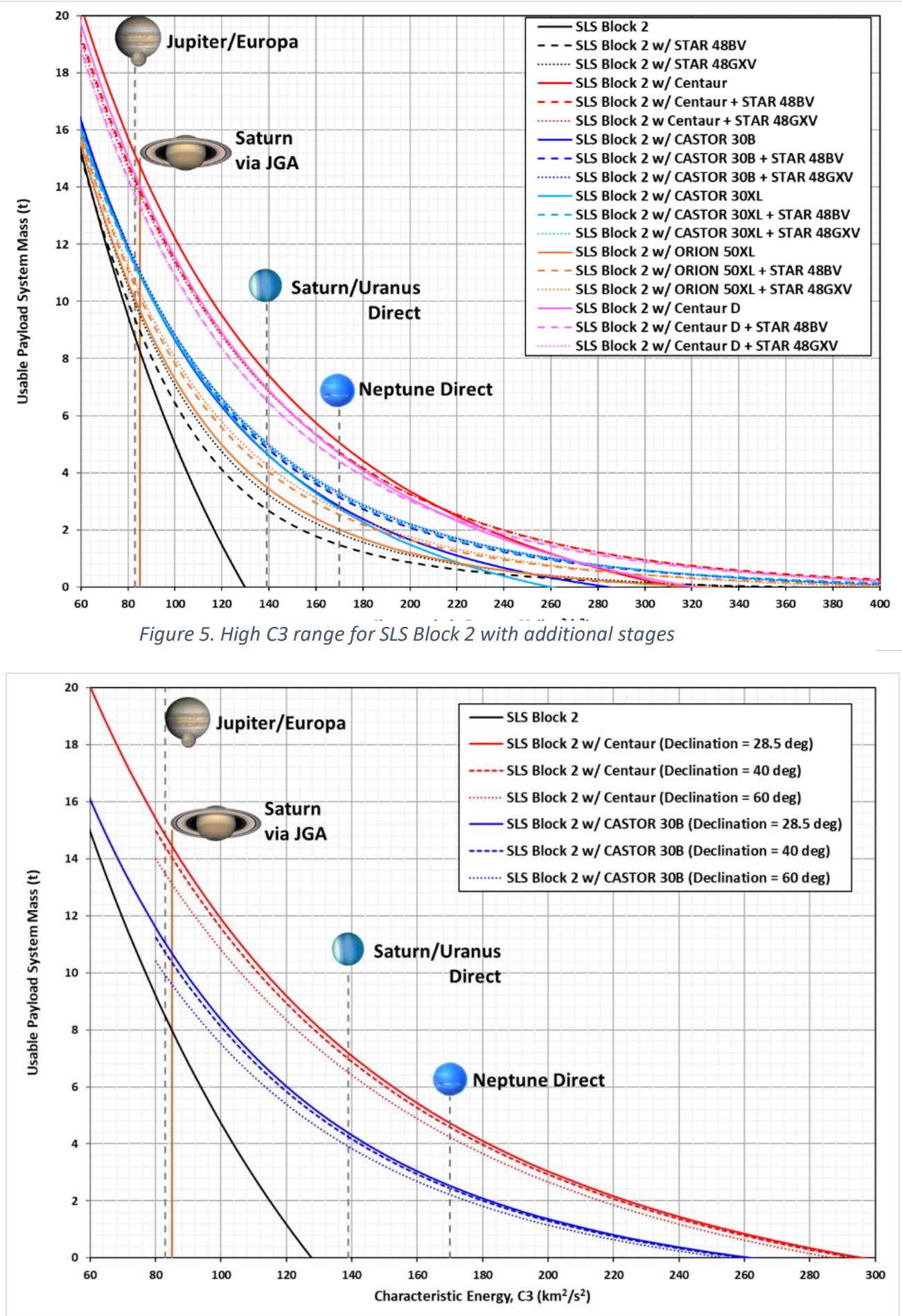

Figure 6. Higher declination C3 performance for both Centaur and CASTOR 3OB third stages

As shown in Figures 4 through 6, the highest payload mass delivered to high $\mathrm{C} 3$ ranges involves LH2/LOX third stages. Adding an LH2/LOX third stage provided an increase from $8 \mathrm{t}$ to $15 \mathrm{t}$ of payload mass delivered to a $\mathrm{C} 3$ of $83 \mathrm{~km} 2 / \mathrm{sec}^{2}$, thus effectively enabling SLS to double the mass that can be sent on a direct trajectory to Jupiter. Moreover, the highest C 3 on record is for the New 
Horizons spacecraft is $157 \mathrm{~km} 2 / \mathrm{s} 2$. If a spacecraft with a mass similar to New Horizons $(\sim 0.8 \mathrm{t})$ were to launch on an SLS Block 2 with a Centaur third stage and a Star 48BV fourth stage, the resulting C3 is greater than $300 \mathrm{~km} 2 / \mathrm{s} 2$-- nearly double the New Horizons mission (Figure 6). Another option would be to increase the payload mass to a C3 of $157 \mathrm{~km} 2 / \mathrm{s} 2$ up to about $6 \mathrm{t}$..

Multiple mission concept teams have identified the payload stage capability of SLS as beneficial for science missions to the outer solar system and beyond. The Interstellar Probe team at Johns Hopkins University's Applied Physics Laboratory (APL) has identified SLS Block 2 with additional third/fourth stages as enabling for launching a New Horizons-class spacecraft beyond the heliosphere with a transit time approaching a decade, depending on mission profile. The configuration offers trade space for multiple trajectory profiles, including a passive Jovian flyby, a powered Jovian flyby, and an Oberth solar gravity assist. This rapid transit to the heliospheric boundary, a fraction that of the Voyager missions, would increase both the operational life of the spacecraft in the interstellar medium and the distance it could reach during that time. [3]

The Neptune-Triton Odyssey team at APL has noted that this capability opens any-year launch opportunities to the Neptune system and reduces velocity prior to Neptune Orbital Insertion, reducing mission risk and increasing available science payload mass. [4] In studying concepts for the Persephone Pluto orbiter mission, members of the New Horizons team at Southwest Research Institute have identified an SLS/Centaur configuration as enabling, given the large mass required by a spacecraft that could both enter and leave the orbit of Pluto. [5] Likewise, the Enceladus Orbilander concept has also studied potential benefits from a multi-stage SLS configuration, notably reduced transit time from an enabled direct transit to Jupiter for a gravity assist to the Saturn system. [6]

The Europa Lander mission concept assumes launch on a baseline SLS Block 1B vehicle in order to support the large launch mass of the payload - more than double any previous planetary science mission [7], but initial analysis indicates a multistage configuration could provide margin for mass or transit time trades.

Multiple studies of planetary defense architectures have shown SLS as enhancing or enabling for mitigation of Potentially Hazardous Objects (PHO), including a 2018 Aerospace Corporation study [8] and the HAMMER concept study [9]. More recently, an initial and so-far-unpublished study at NASA's Goddard Space Flight Center looked at what could be required to divert a short-warning PHO, such as Comet Siding Spring, which was detected only about 22 months before a close flyby of Mars. With only a slightly different orbit, Comet Siding Spring could have impacted Earth. The GSFC study showed that launch C3s of well over $100 \mathrm{~km} 2 / \mathrm{s} 2$ were required for a space mission to fly-by this hypothesized comet in time to mitigate its threat by using a nuclear explosive device (NED) that would alter the orbit of either the comet or its fragments after being blown apart. The required mass of the NED to divert the orbit would be dependent on the mass and other properties of the PHO, as well how long before impact it was diverted. This case was chosen because in part because it seemed to represent on upper bound on difficulty, and the initial results indicate that more than one high-C3 SLS could be required. While in principle it is possible to deliver any given mitigation payload with a larger number of smaller vehicles, the complexity and cost of doing this, particularly when taking schedule into account, favor using the smallest-possible number of the highest-performance vehicle. Even in the cases of PHOs for which there are much longer warning periods, the large required mass of diversion payloads could make the SLS a requirement for developer. 


\section{Progress to First Flight and Beyond}

The initial Block 1 vehicle is completely manufactured and all the elements are at KSC with the exception of the core stage, which at the time of this writing is undergoing "Green Run" testing. After a four-engine hot-firing as part of that test campaign, the stage will be refurbished and shipped KSC. With the five-segment solid rocket booster beginning assembly in June 2020, stacking of the vehicle inside the Vehicle Assembly Building (VAB) will commence with arrival of the core stage from SSC following Green Run. Following assembly, the vehicle will undergo modal testing and a wet dress rehearsal prior to launch, which is scheduled for late 2021. Follow the progress of SLS on the SLS web page or on SLS social media accounts. As NASA's plans for the Artemis program continue to mature, the SLS vehicle remains a cornerstone capability for those evolving plans, with planning manifests showing at least an annual cadence of launches beginning in the mid-2020s, and more flights will be needed as the focus shifts to the first steps on Mars. NASA has shown its commitment to SLS evolution with a series of contracts for manufacturing the vehicle's elements over the next decade. A contract is in place for long-lead items for boosters through the ninth launch of SLS, with a full contract expected next year. Contracts are in place for RS-25 engines through the tenth SLS launch, and NASA has announced that a contract is being negotiated for stages through the twelfth launch.

\section{Conclusions and Recommendations}

SLS is designed to return NASA's human spaceflight program to the Moon and to launch ambitious science missions into the far reaches of the solar system. A flexible system, SLS can be configured to launch the Orion crew vehicle as well as large infrastructure to cislunar space. As the vehicle evolves to progressively more powerful variants, available with $8.4 \mathrm{~m}$ and/or 10 $\mathrm{m}$ diameter payload fairings, opportunities for packaging third and fourth stages with a spacecraft in the large shrouds may be available. Studies show that using these stages (or similar stages available to a mission in the future) with the Block 2 vehicle can be a game-changer for science missions, delivering a new level of ultra-high $\mathrm{C} 3$ performance. It is therefore

\section{recommended that the Decadal Survey prioritize studies to}

- Define the planetary science returns that can be obtained from the existing plans to have baseline SLS versions support the human exploration of the Moon and Mars.

- Identify the statistically most threatening PHO scenarios, as well as envelope the kinds of assets and associated schedules for deploying them that would be required for missions to mitigate these threats.

- Survey how future missions of interest could benefit from high-C3 versions of the SLS from both technical and cost perspectives.

\section{References}

[1] Smith, D. A., "Space Launch System (SLS) Mission Planner's Guide," (2018) [2] Robinson, K. R., et al, "NASA's Space Launch System: Deep Space Access for CubeSats" (2019) [3] Stough, R. W., et al, "NASA's Space Launch System Opens New Frontiers for High C3 Science Missions to Deep Space," AAS 20-646 (2020) [4] McNutt, R. L., et al, "Near-Term Interstellar Probe: First Step," Acta Astronautica Vol 162 pp. 284-299 (2019) [5] Rymer, A., et al, "Neptune-Odyssey: NASA Mission to the Neptune-Triton System," 2020) [6] Howett, C., et al, "Persephone: A Pluto-System Orbiter \& Kuiper Belt Explorer," 2020) [7] MacKenzie, S. M., et al, "Enceladus Orbilander: A Flagship Mission Concept for Astrobiology," (2020) [8] Hand, K., et al., "Europa Lander Mission Concept Update” (2020) [9] Melamed, N., et al, "Planetary Defense Against Asteroid Strikes: Risks, Options, and Costs" 2018) [10] Barbee, B.W., et al, "Options and Uncertainties in Planetary Defense: Mission Planning and Vehicle Design for Flexible Response," Acta Astronautica Vol 143 pp 27-61 (2018) 